\title{
Strain Affects CO Oxidation on Metallic Nanoparticles Non-linearly
}

\author{
Mikkel Jørgensen ${ }^{1}\left[\right.$ ] Henrik Grönbeck ${ }^{1}[0$
}

Published online: 4 March 2019

(c) The Author(s) 2019

\begin{abstract}
Adsorption and reaction energies on metal surfaces are known to depend sensitively on strain. How such effects influence catalytic reactions over nanoparticles is, however, largely unexplored. Here we investigate the effect of strain on the catalytic performance of $\mathrm{CO}$ oxidation over Pt nanoparticles using scaling relations kinetic Monte Carlo simulations. The catalytic activities are compared with the corresponding results for $\operatorname{Pt}(111)$. We find that a moderate expansive strain yields higher catalytic activities for both nanoparticles and extended surfaces. The strong kinetic couplings between different sites on nanoparticles makes the particles respond non-linearly to strain. This is in contrast with $\operatorname{Pt}(111)$, which shows a linear response to strain. The present work demonstrates the possibilities with strain-engineering and highlights the limitation in extrapolating results from extended surfaces to nanoparticles.
\end{abstract}

Keywords Heterogeneous catalysis $\cdot$ Nanoparticles $\cdot$ Strain $\cdot$ Scaling relations $\cdot$ Density functional theory $\cdot$ Kinetic Monte Carlo

\section{Introduction}

Heterogeneous catalysts are generally realized as oxidesupported metal nanoparticles, which typically have a distribution in size and shape. The catalytic performance is determined by the choice of metal, support material, reaction conditions, particle size, and shape. In addition, catalyst performance can be affected by particle strain. Strain in nanoparticles can originate from intrinsic factors such as size, shape [1-4], or from extrinsic factors such as interactions between heterogeneous interfaces [5-8]. Investigating the effects of strain on the catalytic performance of nanoparticles is becoming an increasingly relevant issue as precise measurements and analysis of strain in nanoparticles have been achieved recently [7, 9, 10]. Furthermore, three-dimensional strain maps have also been obtained [11, 12], and strain-engineering has been used to tune catalytic properties in nanoparticles $[6,8,13,14]$.

Mikkel Jørgensen

mikjorge@chalmers.se

Henrik Grönbeck

ghj@chalmers.se

1 Chalmers University of Technology, 41296 Göteborg, Sweden
The number of experimental reports on the relation between strain and catalytic activity is modest. Temperature programmed desorption experiments have been conducted on compressed Pt islands on $\mathrm{Ru}(0001)$, which showed a lower CO desorption temperature as compared to $\mathrm{Pt}(111)$ [15]. Similarly, scanning tunneling microscopy has also shown that $\mathrm{O}$ is destabilized as a consequence of compressive strain [16]. Theoretically, electronic structure calculations predict that both intrinsic and extrinsic strain affect the energetics of catalytic reactions. The results have been rationalized by the d-band model $[17,18]$, which states that the reaction energies are sensitive to the d-band center position, which changes in response to strain.

The $\mathrm{CO}$ oxidation reaction over metallic surfaces has been studied extensively in the past [19]. For this reaction, $\mathrm{Pt}$ is close to the optimal catalyst at relevant reaction conditions [20], and, thus, it is important to investigate whether strain has a significant effect for Pt. Lattice strain effects have been investigated for $\mathrm{CO}$ oxidation over $\mathrm{Pt}(111) \mathrm{using}$ mean-field microkinetic modeling [21]. However, the relation between strain and catalytic activity for nanoparticles remains unexplored. To investigate this, it is crucial to explicitly take nanoparticle effects into account as recently we have shown that kinetic couplings play a major role for the catalytic behavior [10, 22, 23]. 
In this work, we theoretically compare the effect of strain on an extended $\mathrm{Pt}(111)$ surface and Pt nanoparticle. The work is based on density functional theory (DFT) calculations and kinetic Monte Carlo (kMC) simulations. We analyze turnover frequencies, adsorbate coverages, and kinetic couplings for homogeneous strain patterns. We find that a small expansive strain increases the activity for both extended surfaces and nanoparticles, although the responses are different. While strain-effects on extended Pt(111) linearly follow the changes in adsorption energies, the kinetic couplings between sites on nanoparticles cause a non-linear response to strain.

\section{Computational Method}

To simulate the kinetics, we applied the scaling relations Monte Carlo (SRMC) method [10, 24] in the MonteCoffee framework [25]. The SRMC method is outlined below for completeness.

\subsection{Density Functional Theory Calculations}

DFT calculations were performed with the Vienna Ab-Initio Simulation Package (VASP) [26-29] in the ProjectorAugmented Wave (PAW) scheme [30], where the number of valence electrons were: $\mathrm{Pt}(10), \mathrm{O}(6)$ and $\mathrm{C}(4)$. Exchange and correlation was treated in the Generalized Gradient Approximation, with the RPBE [31] functional, which was chosen to get reasonable binding energies for $\mathrm{CO}$ and $\mathrm{O}$. Convergence was addressed using the oxygen chemisorption energy in a $(2 \times 2)$ supercell as a proxy. We tested plane-wave kinetic cutoff, k-point density, and vacuum layer-width. Convergence to within $0.05 \mathrm{eV}$ was observed for a plane-wave cutoff of $450 \mathrm{eV}$, a $(6 \times 6 \times 1) \mathrm{k}$-point grid, and a $12 \AA$ vacuum perpendicular to the slab. The slab was represented by four atomic layers, which was found sufficient for converged surface energies. The Pt lattice constant was determined to be $4.00 \AA$ in the bulk fcc unit-cell, using a $(12 \times 12 \times 12)$ k-point grid.

Structural optimization was performed in the atomistic simulation environment (ASE) [32] with the BFGS LineSearch Algorithm until the maximal force was less than $0.05 \mathrm{eV}^{-1}$. Two bottom layers were fixed to emulate a bulk surface. Vibrational modes were determined in the Harmonic Approximation using two-point finite differences and a displacement of $0.01 \AA$. Adsorbates were considered in singlet spin-states, and adsorption energies were corrected for zero-point motion. Gas-phase molecules were optimized in a $(30 \AA \times 30 \AA \times 30 \AA)$ cell with appropriate spin-states: Singlet $\left(\mathrm{CO}\right.$ and $\left.\mathrm{CO}_{2}\right)$ and triplet $\left(\mathrm{O}_{2}\right)$.

The energy barriers were evaluated using climbing image nudged elastic band (CI-NEB) [33] method from the VTST tools [34]. Seven images were included, and initial interpolations were performed by the Image Dependent Pair Potential method [35]. Transition states were optimized until all forces were lower than $0.05 \mathrm{eV}^{-1}$.

\subsection{Reaction Energy Landscape}

The reaction energy landscape was represented using generalized coordination numbers $[36,37](\overline{\mathrm{CN}})$ as descriptors. $\overline{\mathrm{CN}}$ of a site is an extension of the conventional coordination number that includes the first-nearest-neighbor coordination in a weighted sum:

$\overline{\mathrm{CN}}=\sum_{i} \frac{\mathrm{CN}_{i}}{\mathrm{CN}_{\max }}$

where the sum runs over all nearest neighbors to the site, $\mathrm{CN}_{i}$ is the coordination number of neighbor $i$, and $\mathrm{CN}_{\max }$ is the bulk coordination number of the site. We mapped out the reaction energy landscape by linear fitting of the adsorption energies versus $\overline{\mathrm{CN}}$. This was done using model surfaces (See Supporting Information of [24]). The adsorption energies were fitted as

$E_{\mathrm{CO}}^{\mathrm{ads}}=0.252(\overline{\mathrm{CN}}-7.5)-1.36 \mathrm{eV}$

$E_{\mathrm{O}}^{\mathrm{ads}}=0.218(\overline{\mathrm{CN}}-7.5)-0.95 \mathrm{eV}$

The energy barrier for $\mathrm{CO}_{2}$ formation was fitted to a Brønsted-Evans-Polanyi (BEP) relation as

$\Delta E^{\mathrm{act}}=0.824\left(E_{\mathrm{O}}^{\mathrm{ads}}+E_{\mathrm{CO}}^{\mathrm{ads}}\right)+2.95 \mathrm{eV}$

Adsorbate-adsorbate interactions were implemented as a constant nearest neighbor repulsion, calculated on the (111) surface to be $0.19 \mathrm{eV}$ for $\mathrm{CO}-\mathrm{CO}, 0.32 \mathrm{eV}$ for $\mathrm{O}-\mathrm{O}$, and $0.30 \mathrm{eV}$ for $\mathrm{CO}-\mathrm{O}$ interactions. On the edges and corners, the adsorbate-adsorbate interactions were lowered by a factor $0.5\left(\cos 120^{\circ}\right)$.

Strain was added as a perturbation to the adsorption energies by fitting the adsorption energies of $\mathrm{CO}$ and $\mathrm{O}$ as a function of strain on the (111) surface [10]. The resulting expressions for the perturbation added to the adsorption energies due to strain are:

$\delta E_{\mathrm{CO}}^{\mathrm{ads}}=-0.085 \mathrm{eV} \times \epsilon_{\%}, \quad \delta E_{\mathrm{O}}^{\mathrm{ads}}=-0.105 \mathrm{eV} \times \epsilon_{\%}$

where $\epsilon_{\%}$ is the strain in $\%$, which is positive for expansive strain. Figure 1 shows the adsorption energies of $\mathrm{CO}$ and $\mathrm{O}$ on the (111) surfaces and edges. The difference between adsorption energy on the edge and (111) facets are about $0.6 \mathrm{eV}$ for both $\mathrm{CO}$ and $\mathrm{O}$, and varies with up to $1.0 \mathrm{eV}$ for the considered range of strains. Compression weakens the adsorbate-surface bond, which is in agreement with previous 


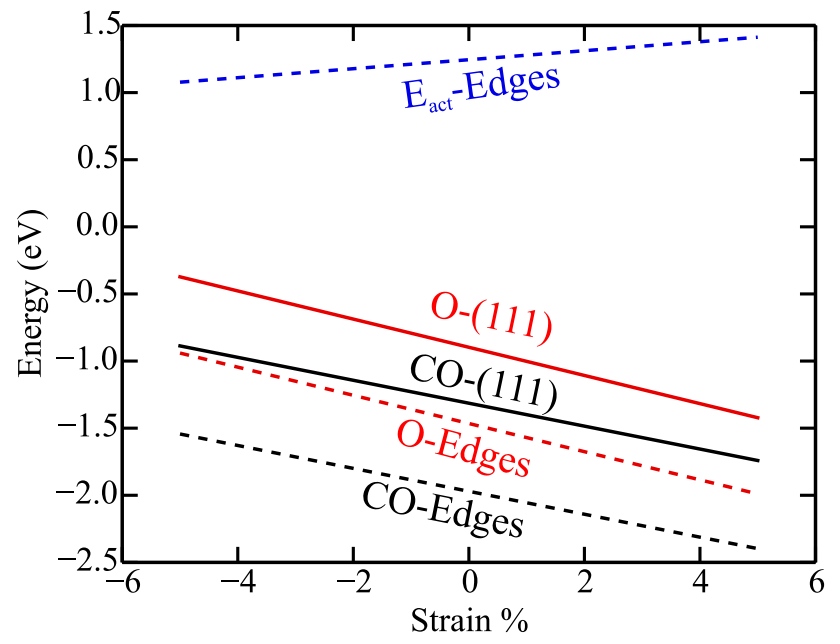

Fig. 1 Dependence of adsorption energies and $\mathrm{CO}_{2}$ formation energy barrier on strain for a $3.5 \mathrm{~nm}$ truncated octahedron. The plotted energies are averaged over $\overline{\mathrm{CN}}$ corresponding to the two different types of sites

theoretical predictions $[18,21,38]$. It is noted that adsorption energies are much more sensitive to adsorbate-adsorbate interactions and the type of site $(\overline{\mathrm{CN}})$ than to strain. The energy barrier for $\mathrm{CO}_{2}$ formation does not scale as strongly with strain as the adsorption energies.

\subsection{Kinetic Monte Carlo}

MonteCoffee [25] uses the first reaction method (FRM) algorithm [39]. In FRM, reactions are executed at randomly generated times, given by the rate constants through

$t_{i, j}=t-\frac{\ln u}{k_{i, j}}$

where $i$ is an index that describes the reaction-type and $j$ is the index of the site where the reaction occurs. $t_{i, j}$ is the time of occurrence of the reaction, $t$ is the simulation-time, $u$ is a random uniform number in [0,1[, and $k_{i, j}$ is the rate-constant of the reaction.

Rate constants for adsorption were calculated using collision theory:

$k_{i, j}^{\text {ads }}=\frac{p_{i} A_{\text {site }} s_{i, j}}{\sqrt{2 \pi M_{i} k_{\mathrm{B}} T}}$

where $i$ denotes the species, $p_{i}$ is the pressure, $A_{\text {site }}=10 \AA^{2}$ is the area of one site, $M_{i}$ is the molecular mass, and $s_{i, j}$ is the sticking coefficient. The sticking coefficients on the facets were set to 0.9 for $\mathrm{CO}$ and 0.1 for $\mathrm{O}_{2}$. On the edges and corners, 1.0 was used for both adsorbates. To ensure thermodynamic consistency, the rate constants for desorption were calculated from the adsorption rates and equilibrium constants as

$k_{i, j}^{\mathrm{des}}=\frac{k_{i, j}^{\mathrm{ads}}}{K_{i, j}}, \quad K_{i, j}=\exp \left(\frac{-\Delta G_{i, j}}{k_{\mathrm{B}} T}\right)$

The rate constants for the $\mathrm{CO}^{*}+\mathrm{O}^{*} \rightarrow \mathrm{CO}_{2}(g)$ reaction were calculated by harmonic transition state theory [40] as:

$k_{i, j}=\frac{k_{\mathrm{B}} T}{h} \exp \left(\frac{-\Delta E_{i, j}^{\mathrm{act}}+T \Delta S_{i, j}^{\mathrm{act}}}{k_{\mathrm{B}} T}\right)$

where $\Delta E_{i, j}^{\text {act }}$ is the energy barrier of the reaction and $\Delta S_{i, j}^{\text {act }}$ is the entropic barrier, evaluated in the harmonic approximation. The energy barrier was calculated on the fly using Eq. (4). Thus, adsorbate-adsorbate interactions affects the barrier through the binding energies. The rate constants for diffusion reactions were also calculated using Eq. (9), where the diffusion barriers are calculated on the (111) surface to be 0.08 and $0.58 \mathrm{eV}$ for $\mathrm{CO}$ and $\mathrm{O}$, respectively. During the kMC simulations, the diffusion rate-constants are adjusted for energy differences between the initial and final states, which ensures thermodynamic consistency. There is a large time-scale separation between diffusion of $\mathrm{CO}$ and the remaining reactions, which was solved by adding $0.45 \mathrm{eV}$ to the $\mathrm{CO}$ diffusion barriers. This approach is reasonable as diffusion remains quasi-equilibrated [39, 41].

In MonteCoffee [25], sites are represented using neighbor-lists between atoms, and each site was treated as a coarse grained entity entailing ontop, bridge, and hollow positions. The Pt(111) surface was simulated on a $14 \times 14$ grid with periodic boundary conditions, resulting in converged TOFs [24]. For the nanoparticles, a truncated octahedron of $3.5 \mathrm{~nm}$ was considered. Constant pressures of $20 \mathrm{mbar}$ $\mathrm{CO}$ and $10 \mathrm{mbar}_{2}$ were applied. The simulations were initialized from $50 \% \mathrm{CO}$-covered surface, and performed until steady-state was sampled sufficiently. To test convergence, 16 identically prepared simulations were performed for each data-point, from which error-bars were calculated.

\section{Results}

\subsection{Turnover Frequencies}

The turnover frequency (TOF) is a common measure of catalytic performance. Figure 2(top) shows the TOF of the extended $\operatorname{Pt}(111)$ surface as a function of temperature. The extended surfaces have TOFs in the range $0-4 \times 10^{3}$ site $^{-1} s^{-1}$, in agreement with experiments [42]. 


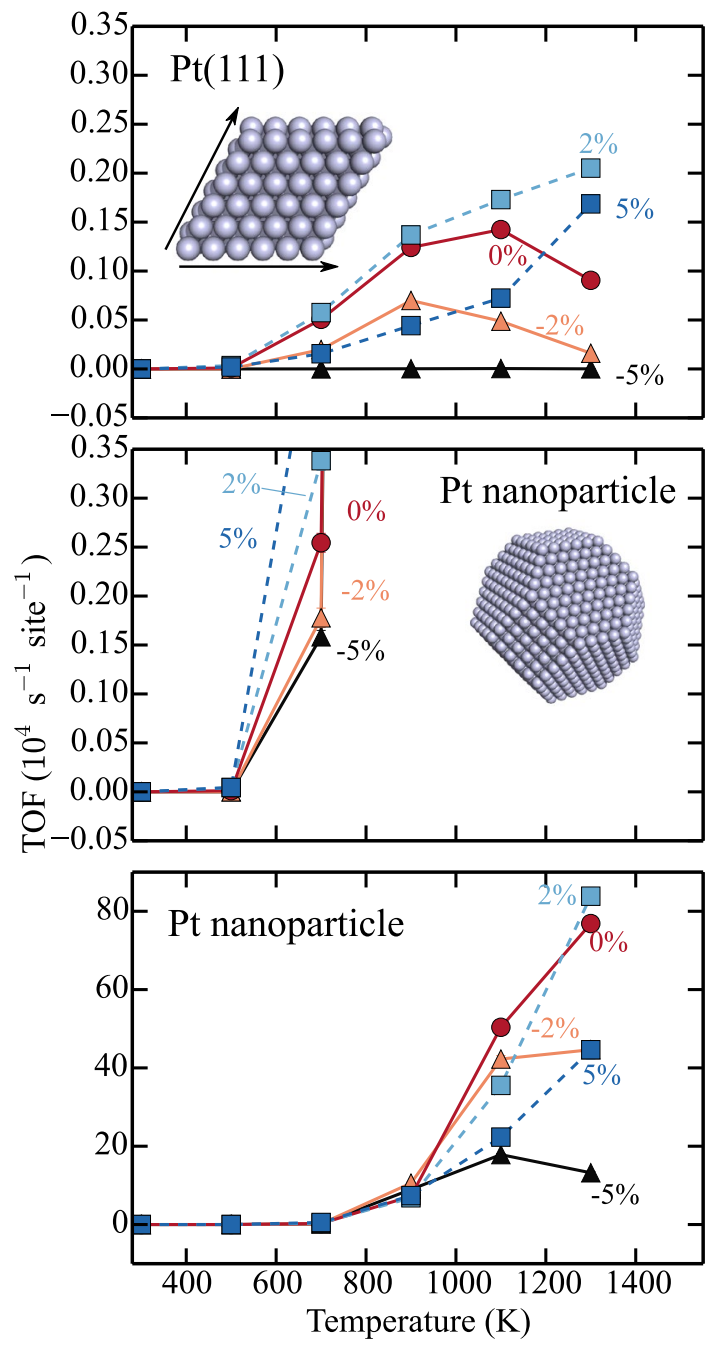

Fig. 2 Turnover frequency for different strains for Pt(111) (top), the Pt nanoparticle at a different scale (middle), and the Pt nanoparticle (bottom). Pressures: 20 mbar $\mathrm{CO}$ and $10 \mathrm{mbar}_{2}$. Error-bars are the standard deviation of the 16 runs

For the unstrained surface, the reaction lights off around $500 \mathrm{~K}$, and the TOF peaks at $1100 \mathrm{~K}$. The $-2 \%$ surface has a similar TOF scaling, however, it peaks at about $900 \mathrm{~K}$. The $-5 \%$ surface has low TOFs in the range $0-4$ site $^{-1} s^{-1}$, with a peak around $1100 \mathrm{~K}$. The $2 \%$ surface yields the largest maximal TOFs of the systems, and does not peak in the studied temperature range. The $5 \%$ surface does not peak in the investigated temperature range. These trends can be understood by the fact that expansive strain binds the adsorbates stronger to the surface, whereas compression lowers the binding strength for both $\mathrm{CO}$ and $\mathrm{O}$. Thus, for compressive strain, the peaks in TOF appear at lower temperatures with lower values. These trends are in full agreement with results from mean-field microkinetic modeling [21].

The TOFs for the Pt nanoparticle are shown in Fig. 2 (middle and bottom). Similar to the surface, for all strain values, the reaction lights off at temperatures close to 500 $\mathrm{K}$. The TOFs range between 0 and $9 \times 10^{5} \mathrm{site}^{-1} \mathrm{~s}^{-1}$. The unstrained particle does not reach a peak activity over the current temperature range. Thus, the TOF is roughly two orders of magnitude higher than for the $\mathrm{Pt}(111)$ surface. The $-2 \%$ particle has a low TOF compared to the unstrained case and peaks after $1300 \mathrm{~K}$. Similarly, the $-5 \%$ particle peaks at $1100 \mathrm{~K}$ and has a low TOF. The $2 \%$ particle does not peak in TOF, and it reaches the highest TOF of all the particles in the investigated temperature range. The 5\% particle has TOFs between the $-2 \%$ particle and the $-5 \%$ particle, and it does not peak in the considered temperature range. The fact that peaks are not observed for the expanded particles is owing to the expansion-induced strengthening of adsorbate binding.

Comparing extended surfaces and nanoparticles, the two systems have similar light-off temperatures. However, nanoparticles are much more active, owing to the kinetic couplings that gives nanoparticles a convoluted response to strain. A further analysis of the kinetic couplings is presented in Sect. 3.3. One reason for the high TOF of the nanoparticle is that the multiple available sites enable $\mathrm{CO}$ and $\mathrm{O}$ to be present simultaneously. Moreover, the binding energies are stronger on low-coordinated sites, which keeps the adsorbates on the surface. This is beneficial as the barrier scales relatively weakly with binding energy (Fig. 1). Although a small expansive strain is beneficial for the activity, both the unstrained nanoparticle and extended surfaces are very active. This suggests that $\mathrm{Pt}$ is close to an optimal catalyst for CO oxidation [20]. However, we note that in reality most sites are strained to some degree $[7,9,10]$.

To further analyze the response to strain, we investigated the TOF of the individual nanoparticle sites, which are reported in Fig. 3. On the (100) and (111) facets, the activity is ordered by strain, where expansion is most beneficial. The TOFs are in the range $0-7 \times 10^{5} \mathrm{site}^{-1} \mathrm{~s}^{-1}$, where the (100) facets are most active. For both types of facets, the light-off temperature is about $500 \mathrm{~K}$. For the edges and corners, there is a less clear trend in the strain, and the light-off occurs at about $800 \mathrm{~K}$. The TOFs on edges and corners are high, up to $1.1 \times 10^{7} \mathrm{site}^{-1} \mathrm{~s}^{-1}$. Comparing the facets to the edges and corners, we see clear qualitative differences. The edges and corners are orders of magnitude more active than facets, which is explained by the higher coverages. A confounding factor 


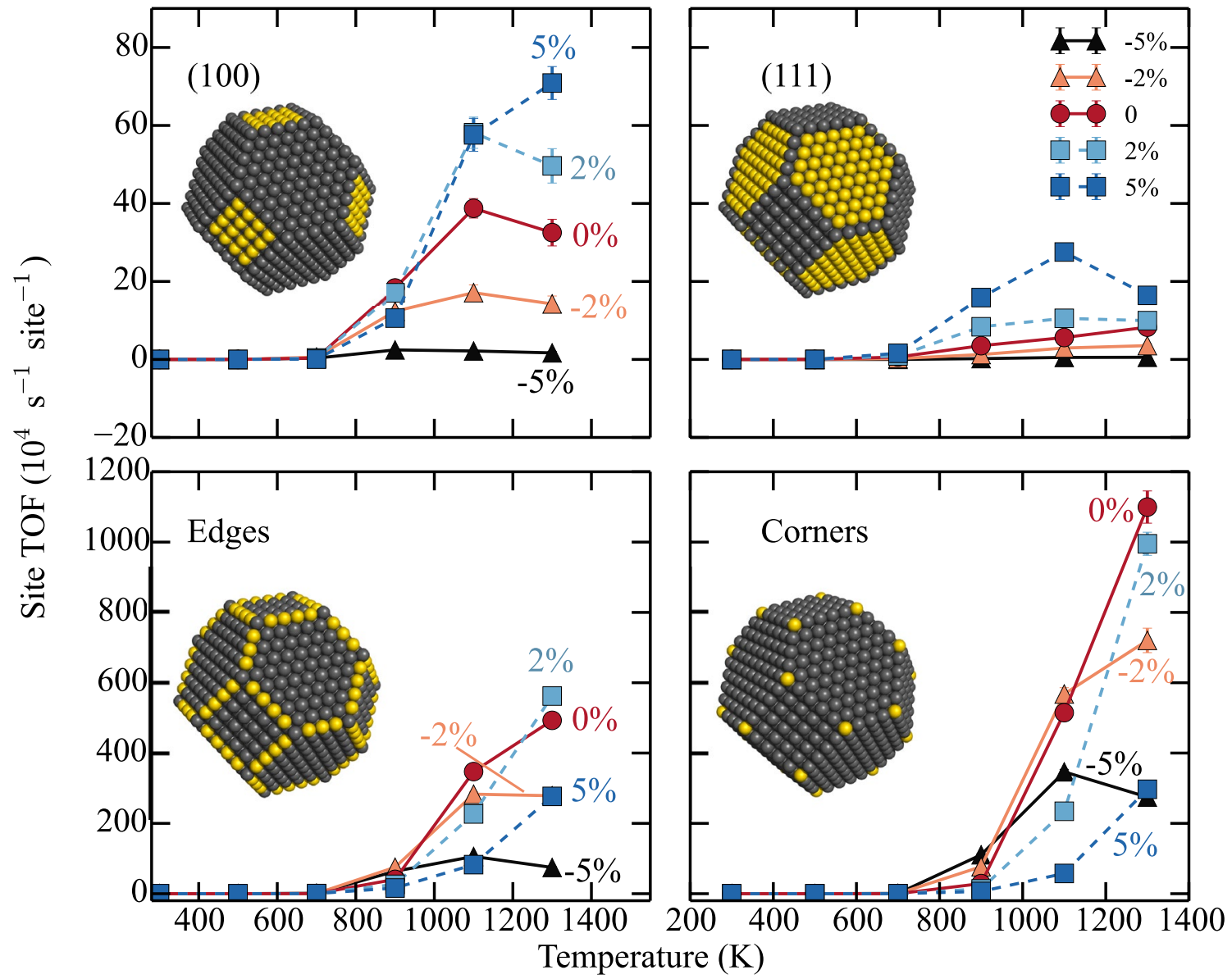

Fig. 3 Turnover frequency of the individual sites on the nanoparticle for different strains. Investigated sites are colored yellow on the particles. Pressures: 20 mbar $\mathrm{CO}$ and 10 mbar $_{2}$. Error-bars are the standard deviation of the 16 runs

is that the adsorbates tend to diffuse towards the edges and corners. Comparing the nanoparticle (111) facet to the extended $\mathrm{Pt}(111)$ surface, the extended surface has an optimal strain value after light-off of $2 \%$, whereas the (111) facet is optimal at $5 \%$. This is due to the different reaction mechanism on the nanoparticle, where $\mathrm{O}_{2}$ dissociates on facets and subsequently diffuses to the edges where $\mathrm{CO}_{2}$ is formed. Thus, the different types of sites respond uniquely to strain, and the total TOF is a non-linear combination of these responses.

\subsection{Surface Coverages}

The average coverages on the extended $\operatorname{Pt}(111)$ surface are shown in Fig. 4. The CO coverages range between 0-0.69 and are highest at low temperatures. For different reasons, the $\mathrm{CO}$ coverage is lowered by both compressive and expansive strain. For compressed surfaces, the lower coverage is caused by weakening of the $\mathrm{CO}$-surface bond. For expansive strain, the increasing $\mathrm{O}$ coverage repels CO. Upon $\mathrm{O}_{2}$ dissociation, the two $\mathrm{O}$ adsorbates separate, and since recombination is unlikely, eventually $\mathrm{O}$ will overtake the surface. The $\mathrm{O}$ coverages range between $0-0.37$, where more expansion results in a higher peakcoverage, owing to the stronger binding energy. Thus, in contrast to $\mathrm{CO}$ coverages, pure thermodynamics governs $\mathrm{O}$ coverages.

On the nanoparticle, the multiple sites render coverages more complex. Figure 5 shows the coverages on the nanoparticle. For the (111) facets, the $\mathrm{CO}$ coverages are highest for expansive strains. The 5\% surface has a irregular increase at $1100 \mathrm{~K}$, which is a feature of the kinetic couplings (See Sect. 3.3). Similarly, the O coverage on the (111) facet is largest for expansive strain. The 

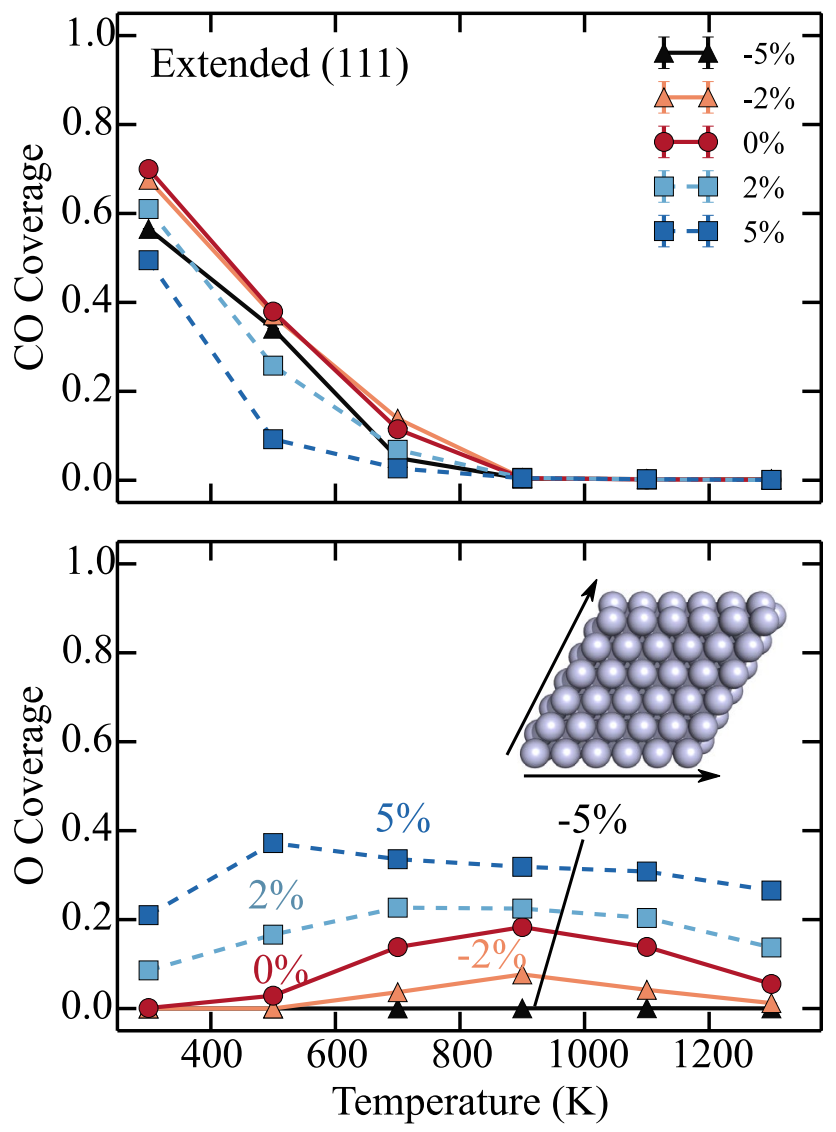

Fig. 4 Average coverages on the extended $\mathrm{Pt}(111)$ surface for $\mathrm{CO}$ (top) and $\mathrm{O}$ (bottom) shown as a function of temperature. Pressures: 20 mbar $\mathrm{CO}$ and $10 \mathrm{mbar}_{2}$. Error-bars are the standard deviation of the 16 runs

edges have a high CO-coverage at low temperatures, and above ca $500 \mathrm{~K}$ there is a finite $\mathrm{O}$ coverage. For edges, both compression and expansion can give higher $\mathrm{CO}$ and $\mathrm{O}$ coverages, which is due to the same mechanisms as described for the (111) facet. Furthermore, the CO desorption temperature is lowered for compressive strain, which is consistent with temperature programmed desorption experiments [15].

For the extended surface, the ratio of $\mathrm{CO} / \mathrm{O}$ adsorption energy explains the response of coverages to strain. In contrast, on the nanoparticle the coverages are affected by finite size effects, thermodynamics driving adsorbates towards the edges, the kinetic couplings between different sites, and the large TOF of the edges. On the nanoparticle facet, the coverages are higher than on the extended surface. This can be attributed to the slightly lower-coordinated perimeter facet sites, and the finite size of the facet.

\subsection{Kinetic Couplings}

The difference in kinetics between nanoparticles and the extended $\mathrm{Pt}(111)$ surfaces is explained by kinetic couplings. To investigate these couplings, we simulated a fictitious nanoparticle (111) facet, where all other sites on the particle were disabled. This facet we shall refer to as the isolated (111) facet. The disabled sites were initiated and kept unoccupied to avoid adsorbate-adsorbate interactions with the active (111) facets. Thus, all adsorption, desorption and diffusion proceed solely on the isolated (111) facet. The isolated facet is different from the extended surface, since it is finite in size and has a lower generalized coordination number on the perimeter.

Figure 6 (top) shows the TOF of the isolated (111) facet. The TOF varies between 0 and $34 \times 10^{4} \mathrm{site}^{-1} s^{-1}$, and the reaction starts at $500 \mathrm{~K}$. The TOF is ordered by strain, where expansion is most favorable. This trend is identical to the extended (111) surface (Fig. 2). However, the isolated facet is more active, partly owing to its finite size that modifies the adsorbate-adsorbate interactions. Another factor is that the perimeter sites on the isolated facet binds the adsorbates stronger. Comparing the isolated facet to the nanoparticle (111) facet (Figs. 6 vs 3), the isolated facet is more active than the nanoparticle facet. This is owing to the edges that take over some part of the reaction on the full nanoparticle.

The CO coverage is shown in Fig. 6 (middle), where the values range between 0 and 0.95 . The compressed surfaces yield the lowest coverages, and the expanded surfaces the highest. This trend is consistent with the extended surface. However, compared to the extended surface, the $\mathrm{CO}$ coverages on the isolated facet are slightly higher. This is owing to the lower-coordinated perimeter sites with reduced adsorbate-adsorbate interactions. In contrast, on the coupled nanoparticle facet, the $\mathrm{CO}$ coverages are more disordered and slightly lower as $\mathrm{CO}$ can diffuse to the edges. The $\mathrm{O}$ coverages are shown in Fig. 6 (bottom), where the values range between 0 and 0.18 . The largest peak coverages are attained for the expanded facets, and the lowest for compressed facets. This trend is similar to the extended surface, however, the finite size of the isolated facet results in more complex coverages, and the peak coverages are 


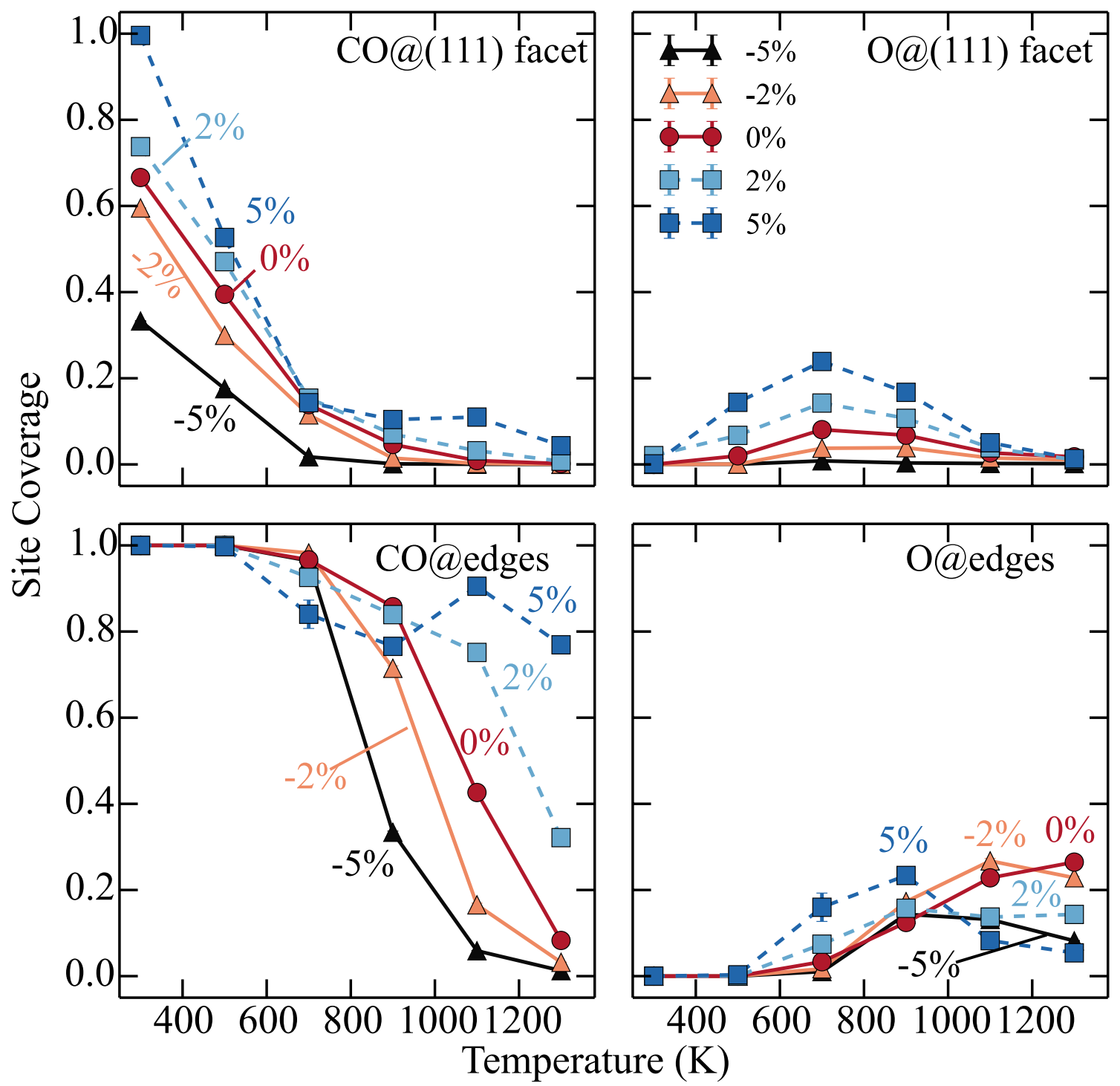

Fig. 5 Average coverage of CO (left) and O (right) for the nanoparticle's (111)-facets (top) and edges (bottom) shown as a function of temperature. Pressures: 20 mbar $\mathrm{CO}$ and $10 \mathrm{mbar}_{2}$. Error-bars are the standard deviation of the 16 runs

smaller. Compared to the coupled nanoparticle facet, the O coverages are very similar at low temperatures, however, at higher temperatures, the isolated facet has a $15 \%$ higher coverage. This is owing to $\mathrm{O}$ diffusion from the facet to the edges on the coupled nanoparticle facet. Despite the small differences in coverages and TOFs, the kinetic couplings and individual sites on the nanoparticle render the effect of strain significantly different on extended surfaces and nanoparticles.

\section{Conclusion}

Here we use scaling relations kinetic Monte Carlo simulations to investigate how nanoparticles and $\mathrm{Pt}(111)$ respond to homogeneous strain. As a model reaction, we investigate $\mathrm{CO}$ oxidation. We find that strain affects nanoparticles and extended surfaces qualitatively differently. This is due to presence of multiple sites on nanoparticles, which mediate kinetic couplings. A slight expansive strain is found to enhance the catalytic activity. Moreover, while thermodynamics rationalizes the adsorbate coverages on extended 

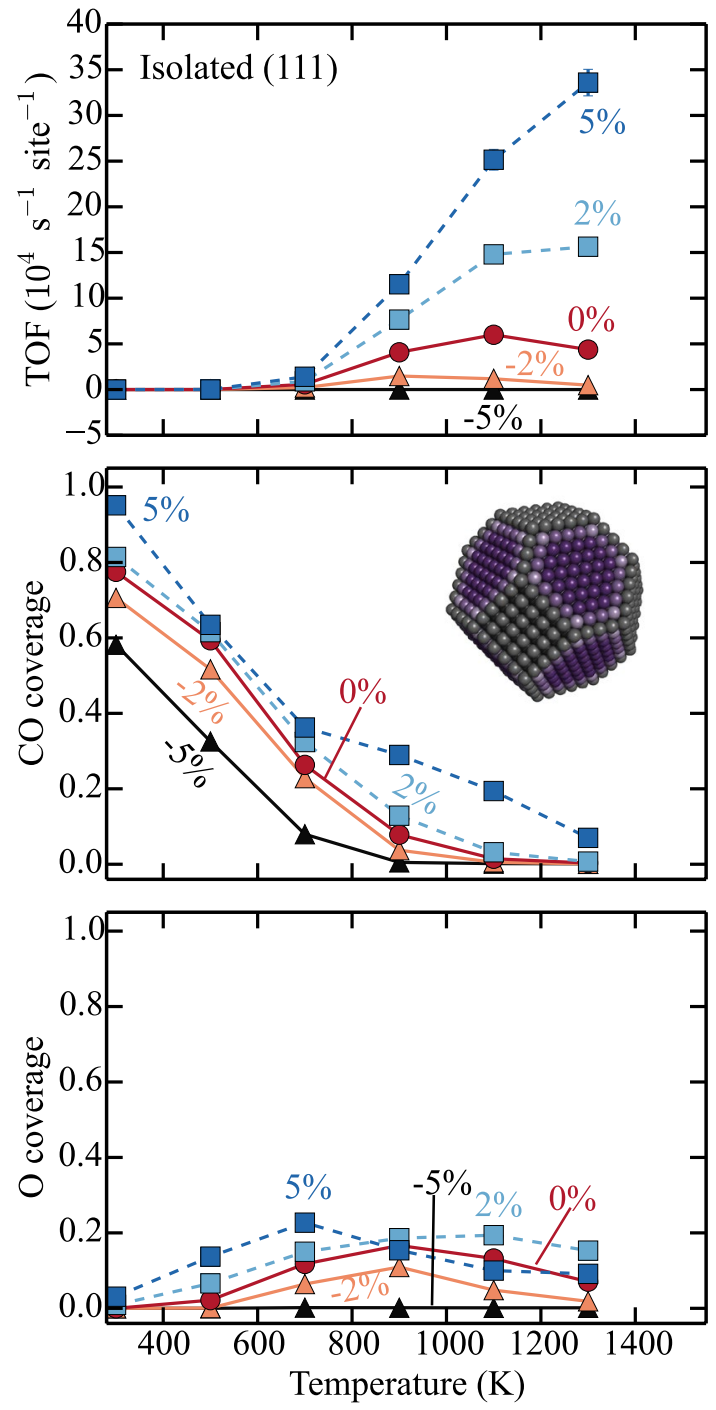

Fig. 6 Isolated (111) facet simulations, where gray sites on the shown particle are disabled. The TOF of the isolated facet (top), the CO coverage on the isolated facet (middle), the $\mathrm{O}$ coverage on the isolated facet (bottom). Pressures: $20 \mathrm{mbar} \mathrm{CO}$ and $10 \mathrm{mbar}_{2}$. Error-bars are the standard deviation of the 16 runs

surfaces, it is insufficient as a measure to understand straineffects on nanoparticles. Although strain influences the activity of nanoparticles, it should be realized that other factors could be more important, including shape, size, and reaction conditions.

We expect kinetic couplings to affect a range of different catalytic reactions. This suggests that care must be taken when extrapolating strain-activity relations from extended surface models to nanoparticles. By explicitly exploring nanoparticles, we have taken an important step towards bridging the materials gap in heterogeneous catalysis, also with respect to strain.
Acknowledgements Financial support is acknowledged from Chalmers Excellence Initiative Nano, and the Swedish Research Council (201605234). The calculations were performed at PDC (Stockholm) and C3SE (Göteborg) via a SNIC grant. The Competence Centre for Catalysis is hosted by Chalmers University of Technology and financially supported by the Swedish Energy Agency and the member companies AB Volvo, ECAPS AB, Johnson Matthey AB, Preem AB, Scania CV $\mathrm{AB}$, Umicore AG \& Co. KG and Volvo Car Corporation AB.

Open Access This article is distributed under the terms of the Creative Commons Attribution 4.0 International License (http://creativeco mmons.org/licenses/by/4.0/), which permits unrestricted use, distribution, and reproduction in any medium, provided you give appropriate credit to the original author(s) and the source, provide a link to the Creative Commons license, and indicate if changes were made.

\section{References}

1. Solliard C, Flueli M (1985) Surf Sci 156:487. https://doi. org/10.1016/0039-6028(85)90610-7

2. Huang WJ, Sun R, Tao J, Menard LD, Nuzzo RG, Zuo JM (2008) Nat Mater 7:308

3. Walsh MJ, Yoshida K, Kuwabara A, Pay ML, Gai PL, Boyes ED (2012) Nano Lett 12:2027. https://doi.org/10.1021/n1300067q

4. Wu J, Qi L, You H, Gross A, Li J, Yang H (2012) J Am Chem Soc 134:11880. https://doi.org/10.1021/ja303950v

5. Strasser P, Koh S, Anniyev T, Greeley J, More K, Yu C, Liu Z, Kaya S, Nordlund D, Ogasawara H, Toney MF, Nilsson A (2010) Nat Chem 2:454. https://doi.org/10.1038/NCHEM.623

6. Zhang S, Zhang X, Jiang G, Zhu H, Guo S, Su D, Lu G, Sun S (2014) J Am Chem Soc 136:7734. https://doi.org/10.1021/ja503 0172

7. Daio T, Staykov A, Guo L, Liu J, Tanaka M, Lyth SM, Sasaki K (2015) Sci Rep 5:13126. https://doi.org/10.1038/srep13126

8. Wang H, Xu S, Tsai C, Li Y, Liu C, Zhao J, Liu Y, Yuan H, Abild-Pedersen F, Prinz FB, Nørskov JK, Cui Y (2016) Science 354:1031

9. Madsen J, Liu P, Wagner JB, Hansen TB, Schiøtz J (2017) Adv Struct Chem Imaging 3:14. https://doi.org/10.1186/s4067 9-017-0047-0

10. Nilsson Pingel T, Jørgensen M, Yankovich A, Grönbeck H, Olsson E (2018) Nat Commun 9:2722

11. Goris B, De Beenhouwer J, De Backer A, Zanaga D, Batenburg KJ, Snchez-Iglesias A, Liz-Marzn LM, Van Aert S, Bals S, Sijbers J, Van Tendeloo G (2015) Nano Lett 15:6996. https://doi.org/10.1021/ acs.nanolett.5b03008

12. Van Aert S, De Backer A, Martinez GT, den Dekker AJ, Van Dyck D, Bals S, Van Tendeloo G (2016) Int Union Crystallogr Monogr Crystallogr 3:71. https://doi.org/10.1107/S2052252515019727

13. Escudero-Escribano M, Malacrida P, Hansen MH, Vej-Hansen UG, Velzquez-Palenzuela A, Tripkovic V, Schiøtz J, Rossmeisl J, Stephens IEL, Chorkendorff I (2016) Science 352:73. https://doi. org/10.1126/science.aad8892

14. Hernandez-Fernandez P, Masini F, McCarthy DN, Strebel CE, Friebel D, Deiana D, Malacrida P, Nierhoff A, Bodin A, Wise AM, Nielsen JH, Hansen TW, Nilsson A, Stephens IEL, Chorkendorff I (2014) Nat Chem 6:732. https://doi.org/10.1038/NCHEM.2001

15. Buatier de Mongeot F, Scherer M, Gleich B, Kopatzki E, Behm RJ (1998) Surf Sci 411:249. https://doi.org/10.1016/S0039 -6028(98)00286-6

16. Gsell M, Jakob P, Menzel D (1998) Science 280:717. https://doi. org/10.1126/science.280.5364.717 
17. Hammer B, Nørskov JK (2002) Nature 376:238. https://doi. org/10.1038/376238a0

18. Mavrikakis M, Hammer B, Nørskov JK (1998) Phys Rev Lett 18:2819

19. van Spronsen MA, Frenken JWM, Groot IMN (2017) Chem Soc Rev 46:4347. https://doi.org/10.1039/C7CS00045F

20. Falsig H, Hvolbæk B, Kristensen IS, Jiang T, Bligaard T, Christensen CH, Nørskov JK (2008) Angew Chem Int Ed 47:4835. https ://doi.org/10.1002/anie.200801479

21. Grabow L, Xu Y, Mavrikakis M (2006) Phys Chem Chem Phys 8:3369. https://doi.org/10.1039/b606131a

22. Jørgensen M, Grönbeck H (2018) Angew Chem Int Ed 57:5086. https://doi.org/10.1002/anie.201802113

23. Zhdanov VP, Kasemo B (1998) Surf Sci 405:27. https://doi. org/10.1016/S0039-6028(97)01078-9

24. Jørgensen M, Grönbeck H (2017) ACS Catal 7:5054. https://doi. org/10.1021/acscatal.7b01194

25. Jørgensen M, Grönbeck H (2018) J Chem Phys 149:114101. https ://doi.org/10.1063/1.5046635

26. Kresse G, Furthmuller J (1996) Phys Rev B 54:11169. https://doi. org/10.1103/PhysRevB.54.11169

27. Kresse G, Furthmuller J (1996) Comput Mater Sci 6:15. https://doi. org/10.1016/0927-0256(96)00008-0

28. Kresse G, Hafner J (1993) Phys Rev B 47:558. https://doi. org/10.1103/PhysRevB.47.558

29. Kresse G, Joubert D (1999) Phys Rev B 59(3):1758. https://doi. org/10.1103/PhysRevB.59.1758

30. Blöchl P (1994) Phys Rev B 50:17953. https://doi.org/10.1103/ PhysRevB.50.17953

31. Hammer B, Hansen LB, Nørskov JK (1999) Phys Rev B 59:7413. https://doi.org/10.1103/PhysRevB.59.7413
32. Bahn SR, Jacobsen KW (2002) Comput Sci Eng 4:56. https://doi. org/10.1109/5992.998641

33. Henkelman G, Uberuaga BP, Jónsson H (2000) J Chem Phys 113:9901. https://doi.org/10.1063/1.1329672

34. Henkelman Group at the University of Texas at Austin Home Page. http://theory.cm.utexas.edu. Accessed Nov 16, 2015

35. Smidstrup S, Pedersen A, Stokbro K, Jónsson H (2014) J Chem Phys 140:214106. https://doi.org/10.1063/1.4878664

36. Calle-Vallejo F, Marnez JI, Garca-Lastra JM, Sautet P, Loffreda D (2014) Angew Chem Int Ed 53:8316. https://doi.org/10.1002/ anie. 201402958

37. Calle-Vallejo F, Loffreda D, Koper MTM, Sautet P (2015) Nat Chem 7:403. https://doi.org/10.1038/nchem.2226

38. Xue T, Wu C, Ding X, Sun J (2018) Phys Chem Chem Phys 20:17927. https://doi.org/10.1039/c8cp01966e

39. Jansen APJ (2012) An introduction to kinetic Monte Carlo simulations of surface reactions. Springer, Berlin, pp 53, 162-163. https:// doi.org/10.1007/978-3-642-29488-4

40. Eyring H (1935) Chem Rev 17:65. https://doi.org/10.1021/cr600 $56 \mathrm{a} 006$

41. Stamatakis M, Vlachos DG (2011) Comput Chem Eng 35:2602. https://doi.org/10.1016/j.compchemeng.2011.05.008

42. Krick Calderon S, Grabau M, Ovari L, Kress B, Steinrück HP, Papp C (2016) J Chem Phys 144:044706. https://doi.org/10.1063/1.49403 18

Publisher's Note Springer Nature remains neutral with regard to jurisdictional claims in published maps and institutional affiliations. 